\title{
The Lattice QCD Study of the Three-Nucleon Force
}

\author{
Takumi Doi (for HAL QCD Collaboration)
}

\author{
Center for Nuclear Study, The University of Tokyo, Tokyo 113-0033, Japan
}

\begin{abstract}
We investigate three-nucleon forces (3NF) from lattice QCD simulations, utilizing the Nambu-Bethe-Salpeter (NBS) wave function to determine two-nucleon forces (2NF) and $3 \mathrm{NF}$ on the same footing. Quantum numbers of the threenucleon $(3 \mathrm{~N})$ system are chosen to be $\left(I, J^{P}\right)=\left(1 / 2,1 / 2^{+}\right)$(the triton channel). We consider the simplest geometrical configuration where $3 \mathrm{~N}$ are aligned linearly with an equal spacing, to reduce the enormous computational cost. Lattice QCD simulations are performed using $N_{f}=2$ dynamical clover fermion configurations at the lattice spacing of $a=0.156 \mathrm{fm}$ on a $16^{3} \times 32$ lattice with a large quark mass corresponding to $m_{\pi}=1.13 \mathrm{GeV}$. We find repulsive $3 \mathrm{NF}$ at short distance.
\end{abstract}

Keywords: Lattice QCD, Nuclear Forces, Three-Nucleon Forces

PACS: 12.38.Gc, 13.75.Cs, 21.30.-x, 21.45.Ff

\section{INTRODUCTION}

One of the hottest topic in nuclear physics and astrophysics these days is the understanding of the properties of 3NF. Actually, there are various phenomena where $3 \mathrm{NF}$ may play an important role, e.g., the binding energies of light nuclei [1], the properties of neutron-rich nuclei and the supernova nucleosynthesis [2] and the nuclear equation of state $(\mathrm{EoS})$ at high density relevant to the physics of neutron stars [3, 4].

Despite of its phenomenological importance, microscopic understanding of $3 \mathrm{NF}$ is still limited. Pioneered by Fujita and Miyazawa [5], $3 \mathrm{NF}$ have been commonly studied from two-pion exchange ( $2 \pi \mathrm{E})$ models with the $\Delta$-excitation. However, since $3 \mathrm{NF}$ is originated by the fact that a nucleon is not a fundamental particle, it is most desirable to determine $3 \mathrm{NF}$ from the fundamental degrees of freedom (DoF), i.e., quarks and gluons, on the basis of quantum chromodynamics (QCD). In this proceeding, we report the calculation of 3NF from first-principle lattice QCD.

As for the 2NF from lattice QCD, an approach based on the NBS wave function has been proposed [6, 7]. Resultant (parity-even) 2NF in this approach are found to have attractive wells at long and medium distances and central repulsive cores at short distance. The method has been extended to the hyperon-nucleon (YN) and hyperon-hyperon (YY) interactions $[8,9,10,11,12]$. In this report, we extend the method to the $3 \mathrm{~N}$ system, and perform the lattice QCD simulations of $3 \mathrm{NF}$ in the triton channel, $\left(I, J^{P}\right)=\left(1 / 2,1 / 2^{+}\right)[13,14,15]$. For details of this study, refer to Ref. [15].

\section{FORMALISM}

Since the detailed formulation for the $2 \mathrm{NF}$ is given in Ref. [7], we discuss the extension to the $3 \mathrm{~N}$ system. We consider the NBS wave function $\psi_{3 N}(\vec{r}, \vec{\rho})$ extracted from the six-point correlator as

$$
\begin{aligned}
G_{3 N}\left(\vec{r}, \vec{\rho}, t-t_{0}\right) & \equiv \frac{1}{L^{3}} \sum_{\vec{R}}\left\langle 0\left|\left(N\left(\vec{x}_{1}\right) N\left(\vec{x}_{2}\right) N\left(\vec{x}_{3}\right)\right)(t) \overline{\left(N^{\prime} N^{\prime} N^{\prime}\right)}\left(t_{0}\right)\right| 0\right\rangle \underset{t \gg t_{0}}{\longrightarrow} A_{3 N} \psi_{3 N}(\vec{r}, \vec{\rho}) e^{-E_{3 N}\left(t-t_{0}\right)} \\
\psi_{3 N}(\vec{r}, \vec{\rho}) & \equiv\left\langle 0\left|N\left(\vec{x}_{1}\right) N\left(\vec{x}_{2}\right) N\left(\vec{x}_{3}\right)\right| E_{3 N}\right\rangle, \quad A_{3 N} \equiv\left\langle E_{3 N}\left|\overline{\left(N^{\prime} N^{\prime} N^{\prime}\right)}\right| 0\right\rangle
\end{aligned}
$$

where $E_{3 N}$ and $\left|E_{3 N}\right\rangle$ denote the energy and the state vector of the $3 \mathrm{~N}$ ground state, respectively, $N\left(N^{\prime}\right)$ the nucleon operator in the sink (source), and $\vec{R} \equiv\left(\vec{x}_{1}+\vec{x}_{2}+\vec{x}_{3}\right) / 3, \vec{r} \equiv \vec{x}_{1}-\vec{x}_{2}, \vec{\rho} \equiv \vec{x}_{3}-\left(\vec{x}_{1}+\vec{x}_{2}\right) / 2$ the Jacobi coordinates.

With the derivative expansion of the potentials [16], the NBS wave function can be converted to the potentials through the following Schrödinger equation,

$$
\left[-\frac{1}{2 \mu_{r}} \nabla_{r}^{2}-\frac{1}{2 \mu_{\rho}} \nabla_{\rho}^{2}+\sum_{i<j} V_{2 N}\left(\vec{r}_{i j}\right)+V_{3 N F}(\vec{r}, \vec{\rho})\right] \psi_{3 N}(\vec{r}, \vec{\rho})=E_{3 N} \psi_{3 N}(\vec{r}, \vec{\rho}),
$$


where $V_{2 N}\left(\vec{r}_{i j}\right)$ with $\vec{r}_{i j} \equiv \vec{x}_{i}-\vec{x}_{j}$ denotes the $2 \mathrm{NF}$ between $(i, j)$-pair, $V_{3 N F}(\vec{r}, \vec{\rho})$ the $3 \mathrm{NF}, \mu_{r}=m_{N} / 2, \mu_{\rho}=2 m_{N} / 3$ the reduced masses. If we calculate $\psi_{3 N}(\vec{r}, \vec{\rho})$ for all $\vec{r}$ and $\vec{\rho}$, and if all $V_{2 N}\left(\vec{r}_{i j}\right)$ are obtained by (separate) lattice calculations for genuine $2 \mathrm{~N}$ systems, we can extract $V_{3 N F}(\vec{r}, \vec{\rho})$ through Eq. (3).

In practice, however, the computational cost is enormous, because of enlarged DoF by the $3 \mathrm{~N}$ (i.e., 9 quarks) and factorial number of Wick contractions. In order to reduce the cost, we develop several techniques, e.g., taking advantage of symmetries, and employing the non-relativistic limit for the nucleon operator in the source. We further restrict the geometry of the $3 \mathrm{~N}$. More specifically, we consider the "linear setup" with $\vec{\rho}=\overrightarrow{0}$, with which $3 \mathrm{~N}$ are aligned linearly with equal spacings of $r_{2} \equiv|\vec{r}| / 2$. In this setup, the third nucleon is attached to (1,2)-nucleon pair with only $\mathrm{S}$-wave. Considering the total $3 \mathrm{~N}$ quantum numbers of $\left(I, J^{P}\right)=\left(1 / 2,1 / 2^{+}\right)$, the triton channel, the wave function can be completely spanned by only three bases, which can be labeled by the quantum numbers of $(1,2)$-pair as ${ }^{1} S_{0},{ }^{3} S_{1}$, ${ }^{3} D_{1}$. Therefore, the Schrödinger equation leads to the $3 \times 3$ coupled channel equations with the bases of $\psi_{1} S_{0}, \psi_{{ }^{3} S_{1}}$, $\psi_{3^{3} D_{1}}$. The reduction of the dimension of bases is expected to improve the $\mathrm{S} / \mathrm{N}$ as well.

We then consider the identification of genuine 3NF. It is a nontrivial work: Although both of parity-even and parityodd $2 \mathrm{NF}$ are required to subtract $2 \mathrm{NF}$ part in Eq. (3), parity-odd 2NF have not been obtained yet in lattice QCD. In order to resolve this issue, we consider the following channel,

$$
\psi_{S} \equiv \frac{1}{\sqrt{6}}\left[-p_{\uparrow} n_{\uparrow} n_{\downarrow}+p_{\uparrow} n_{\downarrow} n_{\uparrow}-n_{\uparrow} n_{\downarrow} p_{\uparrow}+n_{\downarrow} n_{\uparrow} p_{\uparrow}+n_{\uparrow} p_{\uparrow} n_{\downarrow}-n_{\downarrow} p_{\uparrow} n_{\uparrow}\right],
$$

which is anti-symmetric in spin/isospin spaces for any $2 \mathrm{~N}$-pair. Combined with the Pauli-principle, it is automatically guaranteed that any $2 \mathrm{~N}$-pair couples with even parity only. Therefore, we can extract $3 \mathrm{NF}$ unambiguously using only parity-even 2NF. Note that no assumption on the choice of 3D-configuration of $\vec{r}, \vec{\rho}$ is imposed in this argument, and we can take advantage of this feature for future $3 \mathrm{NF}$ calculations with various $3 \mathrm{D}$-configuration setup.

\section{LATTICE QCD SETUP AND RESULTS}

We employ $N_{f}=2$ dynamical configurations with mean field improved clover fermion and RG-improved gauge action generated by CP-PACS Collaboration [17]. We use 598 configurations at $\beta=1.95$ and the lattice spacing of $a^{-1}=1.269$ (14) GeV, and the lattice size of $V=L^{3} \times T=16^{3} \times 32$ corresponds to $(2.5 \mathrm{fm})^{3}$ box in physical spacial size. For $u, d$ quark masses, we take the hopping parameter at the unitary point as $\kappa_{u d}=0.13750$, which corresponds to $m_{\pi}=1.13 \mathrm{GeV}, m_{N}=2.15 \mathrm{GeV}$ and $m_{\Delta}=2.31 \mathrm{GeV}$. We use the wall quark source with Coulomb gauge fixing. In order to enhance the statistics, we perform the measurement at 32 source time slices for each configuration, and the forward and backward propagations are averaged. The results from both of total angular momentum $J_{z}= \pm 1 / 2$ are averaged as well. We perform the simulation at eleven physical points of the distance $r_{2}$ with the linear setup.

In Fig. 1 (left), we plot the radial part of each wave function of $\psi_{S}=\left(-\psi_{1} S_{0}+\psi_{3_{S_{1}}}\right) / \sqrt{2}, \psi_{M} \equiv\left(\psi_{1_{S_{0}}}+\psi_{3_{S_{1}}}\right) / \sqrt{2}$ and $\psi_{3} D_{1}$ obtained at $\left(t-t_{0}\right) / a=8$. Here, we normalize the wave functions by the center value of $\psi_{S}\left(r_{2}=0\right)$. What is noteworthy is that the wave functions are obtained with good precision, which is quite nontrivial for the $3 \mathrm{~N}$ system. We observe that $\psi_{S}$ overwhelms the wave function, indicating that higher partial waves are strongly suppressed, and thus the effect of the next leading order in the derivative expansion, spin-orbit forces, is suppressed in this lattice setup.

We determine $3 \mathrm{NF}$ by subtracting $2 \mathrm{NF}$ from total potentials in the $3 \mathrm{~N}$ system. Since we have only one channel (Eq. (4)) which is free from parity-odd 2NF, we can determine one type of 3NF. In this report, 3NF are effectively represented in a scalar-isoscalar functional form, which is often employed for the short-range $3 \mathrm{NF}$ in phenomenology.

In Fig. 1 (right), we plot the results for the effective scalar-isoscalar 3NF at $\left(t-t_{0}\right) / a=8$. Here, we include $r_{2}$ independent shift by energies, $\delta_{E} \simeq 5 \mathrm{MeV}$, which is determined by long-range behavior of potentials (2NF and effective $2 \mathrm{NF}$ in the $3 \mathrm{~N}$ system) [15]. While $\delta_{E}$ suffers from $\lesssim 10 \mathrm{MeV}$ systematic error, it does not affect the following discussions much, since $\delta_{E}$ merely serves as an overall offset. In order to check the dependence on the sink time slice, we calculate $3 \mathrm{NF}$ at $\left(t-t_{0}\right) / a=9$ as well, and confirm that the results are consistent with each other [15].

Fig. 1 (right) shows that $3 \mathrm{NF}$ are small at the long distance region of $r_{2}$. This is in accordance with the suppression of $2 \pi \mathrm{E}-3 \mathrm{NF}$ by the heavy pion. At the short distance region, on the other hand, an indication of repulsive $3 \mathrm{NF}$ is observed. Note that a repulsive short-range $3 \mathrm{NF}$ is phenomenologically required to explain the properties of high density matter. Since multi-meson exchanges are strongly suppressed by the large quark mass, the origin of this short-range $3 \mathrm{NF}$ may be attributed to the quark and gluon dynamics directly. In fact, we recall that the short-range repulsive (or attractive) cores in the generalized two-baryon potentials are calculated in lattice QCD in the flavor SU(3) limit, and the results are found to be well explained from the viewpoint of the Pauli exclusion principle in the quark level [9]. In this context, 

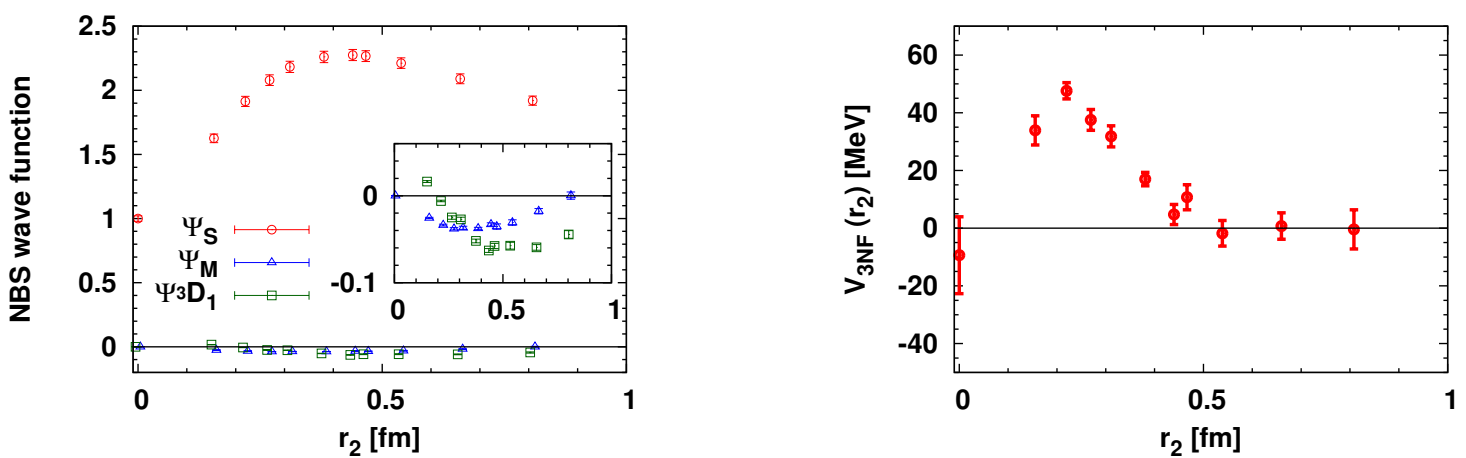

FIGURE 1. (color online). (Left) $3 \mathrm{~N}$ wave functions at $\left(t-t_{0}\right) / a=8$. Circle (red), triangle (blue), square (green) points denote $\psi_{S}, \psi_{M}, \psi^{3} D_{1}$, respectively. (Right) The effective scalar-isoscalar $3 \mathrm{NF}$ in the triton channel with the linear setup.

it is intuitive to expect that the $3 \mathrm{~N}$ system is subject to extra Pauli repulsion effect, which could be an origin of the observed short-range repulsive 3NF. Further investigation along this line is certainly an interesting subject in future.

As regards the systematic error, one may worry about the discretization error, since the nontrivial results are obtained at short distance. In particular, the kinetic terms could suffer from a substantial effect, since they are calculated by the finite difference Laplacian operator as $\nabla^{2} f(x)=\nabla_{\text {std }}^{2} f(x) \equiv \frac{1}{a^{2}} \sum_{i}\left[f\left(x+a_{i}\right)+f\left(x-a_{i}\right)-2 f(x)\right]$. In order to estimate this artifact, we also analyze using the improved Laplacian operator for both of $2 \mathrm{~N}$ and $3 \mathrm{~N}$, $\left.\nabla_{\text {imp }}^{2} f(x) \equiv \frac{1}{12 a^{2}} \sum_{i}\left[-\left(f\left(x+2 a_{i}\right)+f\left(x-2 a_{i}\right)\right)+16\left(f\left(x+a_{i}\right)+f\left(x-a_{i}\right)\right)-30 f(x)\right)\right]$. We observe that the results are consistent with each other, and the discretization artifact of 3NF in Laplacian operator is small [15]. Of course, this study probes only a part of discretization errors, and explicit simulations with a finer lattice are on-going.

Since the lattice simulations are carried out only at single large quark mass, quark mass dependence of $3 \mathrm{NF}$ is certainly an important issue. In the case of $2 \mathrm{NF}$, short-range cores have the enhanced strength and broaden range by decreasing the mass [7]. We, therefore, would expect a significant quark mass dependence exist in short-range $3 \mathrm{NF}$ as well. Quantitative investigation through lattice simulations with lighter quark masses are currently underway.

We thank authors and maintainers of CPS++[18]. We also thank CP-PACS Collaboration and ILDG/JLDG [19] for providing gauge configurations. The numerical simulations have been performed on Blue Gene/L at KEK, T2K at University of Tsukuba and SR16000 at YITP in Kyoto University. This research is supported in part by MEXT Grantin-Aid (20340047, 22540268), Scientific Research on Innovative Areas (20105001, 20105003, 21105515), Specially Promoted Research (13002001), JSPS 21.5985 and HPCI PROGRAM, the Large Scale Simulation Program of KEK (09-23, 09/10-24) and the collaborative interdisciplinary program at T2K-Tsukuba (09a-11, 10a-19).

\section{REFERENCES}

1. S. C. Pieper, Riv. Nuovo Cim. 31, 709 (2008) [arXiv:0711.1500 [nucl-th]].

2. T. Otsuka, T. Suzuki, J. D. Holt, A. Schwenk and Y. Akaishi, Phys. Rev. Lett. 105, 032501 (2010) [arXiv:0908.2607 [nucl-th]].

3. A. Akmal, V. R. Pandharipande and D. G. Ravenhall, Phys. Rev. C58, 1804 (1998) [nucl-th/9804027].

4. S. Nishizaki, T. Takatsuka and Y. Yamamoto, Prog. Theor. Phys. 108, 703 (2002).

T. Takatsuka, S. Nishizaki and R. Tamagaki, Prog. Theor. Phys. Suppl. 174, 80 (2008).

5. J. Fujita and H. Miyazawa, Prog. Theor. Phys. 17, 360 (1957).

6. N. Ishii, S. Aoki and T. Hatsuda, Phys. Rev. Lett. 99, 022001 (2007) [nucl-th/0611096].

7. S. Aoki, T. Hatsuda and N. Ishii, Prog. Theor. Phys. 123, 89 (2010) [arXiv:0909.5585 [hep-lat]].

8. H. Nemura, N. Ishii, S. Aoki and T. Hatsuda, Phys. Lett. B673, 136 (2009) [arXiv:0806.1094 [nucl-th]].

9. T. Inoue et al. [HAL QCD Collab.], Prog. Theor. Phys. 124, 591 (2010) [arXiv:1007.3559 [hep-lat]].

10. K. Sasaki [HAL QCD Collab.], PoS LATTICE2010, 157 (2010) [arXiv:1012.5685 [hep-lat]].

11. T. Inoue et al. [HAL QCD Collab.], Phys. Rev. Lett. 106, 162002 (2011) [arXiv:1012.5928 [hep-lat]].

12. S. Aoki et al. [HAL QCD Collaboration], Proceedings of the Japan Academy, Ser. B, in press, [arXiv:1106.2281 [hep-lat]].

13. T. Doi [HAL QCD Collab.], PoS LATTICE2010, 136 (2010) [arXiv:1011.0657 [hep-lat]].

14. T. Doi [HAL QCD Collab.], Proc. of the Int. Conf. on the structure of baryons (BARYONS'10), [arXiv:1105.6247 [hep-lat]].

15. T. Doi et al. [HAL QCD Collab.], arXiv:1106.2276 [hep-lat].

16. K. Murano, N. Ishii, S. Aoki and T. Hatsuda, Prog. Theor. Phys. 125, 1225 (2011) [arXiv:1103.0619 [hep-lat]].

17. A. Ali Khan et al. [CP-PACS Collab.], Phys. Rev. D 65, 054505 (2002) [E: D 67, 059901 (2003)].

18. Columbia Physics System (CPS), http://qcdoc.phys. columbia.edu/cps.html

19. http://www.lqcd.org/ildg, http://www.jldg.org 